\title{
OPTIMAL SCHEDULING OF A FLEXIBLE-DURATION REST PERIOD FOR A WORK GROUP
}

\author{
STEPHEN E. BECHTOLD \\ Florida State University, Tallahassee, Florida \\ GARY M. THOMPSON \\ University of Utah, Salt Lake City, Utah
}

(Received March 1988; revisions received November 1990, December 1991; accepted March 1992) 


\author{
Abstract \\ All previous modeling research involving optimization of performance associated with work-rest cycles \\ has focused upon individual employees working independently. We extend this earlier research by \\ considering the choices of placement for and duration of a single rest period that must be taken \\ simultaneously by all employees in a work group. Assuming linear work-rate decay and recovery \\ functions for individual employees within the group, we show that an appropriate model can be \\ formulated as a mixed-binary, cubic programming problem. We develop an efficient optimal solution \\ procedure with a computational time that appears to be a linear function of the number of employees \\ considered. We report on a preliminary simulation experiment that evaluates the productivity loss that \\ occurs when the rest break policy is determined based on the work characteristics of a randomly selected \\ subset of employees in the work group, and provides an initial exploration of the nature of optimal policy \\ variables. Finally, we offer suggestions for future research. \\ Subject classifications: Labor: finding the optimal timing and length of a common rest break. \\ Programming, nonlinear algorithms: an efficient algorithm for a mixed-binary cubic problem. \\ Simulation, applications: measuring employee work-group performance. \\ Area of review: Manufacturing, Operations and Scheduling .
}


Lagging productivity growth in the United States is a matter of pressing concern. The incentive exists, therefore, to use every reasonable method to increase employee output. It is well known that different work-rest schedules can result in different productivity levels for individuals engaged in either mental or physical tasks (McFarland 1971, Barnes 1980, Baker, Holding and Loeb 1984).

A major problem, from both a research and a practical perspective, has been with respect to the proper methodology for development of effective work-rest policies that can be described by the number, placement, and duration of rest periods. In practice, work-rest policies are frequently based upon the naive insertion of a break period approximately midway in a 4-5 hour span of work.

The importance of optimizing performance as a function of work-rest schedules has been noted (Lee 1974, Morgan and Pitts 1985), and the efficacy of model-based policies for individual employees has been experimentally supported (Boothe 1984, Janaro and Bechtold 1985). Unfortunately, the vast majority of a large body of related research has focused upon the empirical search for effective policies (Woodward and Nelson 1974, Morgan and Pitts 1985).

All previously published modeling research has dealt with maximizing the output of an individual employee working independently. In this paper, we model the performance of a group of employees subject to a common work-rest schedule, the need for which may arise from organizational policies. Work-rest policy development is more complex for an employee group than for an individual because a policy that is effective for one employee may be ineffective for another.

In Section 1 we review the relevant modeling literature. In Section 2 we show that an appropriate model is a mixed-binary, cubic programming problem, and that it is possible to obtain global optimal solutions by solving a series of quadratic subproblems. Section 3 is devoted to the development and computational evaluation of a simplified global optimal procedure. Section 4 contains a simulation-based evaluation of the efficacy of model-derived policies based on the work characteristics of randomly selected employees in the group. In Section 5, we conclude with a discussion of the results and note relevant issues for further research. 


\section{WORK-REST MODELING LITERATURE}

Eilon (1964) pioneered the concept of modeling performance with the objective of determining the optimal length and placement of one rest period, over a finite time horizon, for a single employee. Gentzler, Khalil and Sivazlian (1977) and Bechtold (1979) developed multirest period models for individuals over infinite and finite time horizons, respectively.

Validity issues inherent in these early models suggested that the nature of performance during work and recovery during rest would have to be included in the modeling process (Bechtold, Janaro and Sumners 1984). Most empirical research has suggested that performance patterns generally exhibit either linear or exponential decay, depending upon task characteristics (McCormack 1958, Aquilano 1968, Levine, Romashko and Fleishman 1973, Hagan, Wilkerson and Noble 1980, Okogbaa 1983, Boothe 1984, Janaro and Bechtold 1985). While recovery patterns cannot be observed directly, evidence exists that suggests they also tend to be linear or exponential, depending upon task characteristics (Murrell 1965, Levine, Romashko and Fleishman 1973, Hagan, Wilkerson and Noble 1980, Boothe 1984).

Bechtold, Janaro and Sumners (1984) developed a multiple rest break model that was designed to address the validity issues associated with earlier models. Assuming linear performance decay during work and linear recovery of work-rate-performance potential during rest, the output of the model provides optimal work-rest policies for individual employees assumed to be working on independent tasks. This model was applied in experimental settings using human subjects by Boothe and by Janaro and Bechtold. Respectively, these researchers observed productivity improvements of approximately $8 \%$ for a mental task and approximately 13\% for a physical task. Bechtold and Sumners (1988) later developed a methodology for multiple rest-break policy determination for individual employees where performance decay is exponential and recovery of work rate potential is linear.

Bechtold (1991) proved that the optimal placement of a set of breaks with exogenously determined durations may result in an employee reaching full recovery before the completion of the rest period. All other work-rest models in the literature have presumed that employees resume work at the 
instant that full recovery is achieved, a valid assumption given that break durations were endogenously determined.

\section{A WORK-REST MODEL FOR AN EMPLOYEE GROUP}

Within this section, we provide useful background information, develop the mixed-binary, cubic programming formulation for work-rest policy determination for employee groups, and show that optimal solutions can be obtained by solving a series of quadratic programming problems.

\subsection{Background}

The models developed in this paper meet the assumptions of the models developed by Bechtold, Janaro and Sumners with a single exception: Any employee can be idle (neither working or recovering) even though full recovery has been attained. While the work output of any idle employees would be greater with a shorter break, their lost work may be more than offset by the gain in work output from those employees who have yet to reach full recovery.

Based upon the categorization presented in Bechtold (1991), the nature of any work-rest policy can, for each employee in the work group, be described by one or more of the following conditions:

1. Less Than Full Recovery - the duration of the break is insufficient to return the employee to full recovery;

2. Exact Full Recovery - the employee achieves full recovery at the instantaneous completion of the break;

3. Inexact Full Recovery — the employee reaches full recovery before the completion of the break;

4. At-Most Full Recovery—-the employee has experienced either less than or exact full recovery;

5. At-Least Full Recovery—-the employee has experienced either exact or inexact full recovery. Hereafter, we also will refer to any employee described by the third condition as being idle. 


\subsection{Development of a Mixed-Binary, Cubic Formulation}

Consider the difference between a full recovery break and a break that does not lead to full recovery

(Figure 1). If the duration of the rest-break results in at least full recovery for the ith employee, then that individual's work rate at the beginning of the second work period is at its maximum, and the employee's

\section{INSERT FIGURE 1 HERE}

Figure 1. Three possible work-rate decay and recovery patterns.

work output is:

$W A L F_{i} \int_{0}^{t}\left(c_{i}-b_{i} \tau\right) d \tau$

$$
+\int_{0}^{T-t-\theta}\left(c_{i}-b_{i} \tau\right) d \tau
$$

Subject to $\alpha_{i} \Theta \geqslant b_{i} t$

where

$c_{i}=$ the initial and maximum (full recovery) work rate for the ith employee (assumed to be constant);

$b_{i}=$ the rate of decay in the work rate for the ith employee (assumed to be constant);

$\alpha_{i}=$ the rate of increase in potential work rate during rest for the $i$ th employee (assumed to be constant);

$\Theta=$ the duration of the rest break;

$t=$ the duration of the initial work period; and

$T=$ the length of the time horizon over which the employees are available for work (exogenously

determined).

Equation 1 is appropriate for the computation of the work output of employees 1 and 2 in Figure 1.

Alternatively, if the break duration results in at most full recovery for the ith employee, then the employee's work output is:

$$
\begin{aligned}
& \operatorname{WAMF}_{i}(T)=\int_{0}^{t}\left(c_{i}-b_{i} \tau\right) d \tau \\
& \quad+\int_{0}^{T-t-\theta}\left(c_{i}-b_{i} t+a_{i} \Theta-b_{i \tau}\right) d \tau
\end{aligned}
$$

subject to $a_{i} \Theta \leqslant b_{i} t$. 
Equation 2 is appropriate for the work output computation of employee 3 in Figure 1.

Though we assume, as did Bechtold, Janaro and Sumners, that the time horizon T is exogenously determined, we represent work output as a function of $\mathrm{T}$ for two reasons. First, the length of the time horizon greatly influences group work output. Second, the duration of the time horizon may be partially under managerial control.

The problem of selecting an optimal, single rest-break policy for multiple employees can be formulated as a mixed-binary, cubic programming problem (MBCP) with linear constraints:

maximize work $=W(T)$

$=\sum_{i \in E}\left[y_{i} W A M F_{i}(T)+\left(1-y_{i} W A L F_{i}(T)\right]\right.$

subject to

$b_{i} t-a_{i} \Theta \leqslant M y_{i} \quad$ for $i \in E$,

$a_{i} \Theta-b_{i} t \leqslant M\left(1-y_{i}\right)$ for $i \in E$,

$t+\theta \leqslant T$,

$t \geqslant 0$

$\Theta \geqslant 0$

$y_{i}=0,1$

for $i \in E$,

where $E$ = the set of employees in the work group, and $\mathrm{M}=$ a large positive integer. Employee $i$ has achieved at least full recovery if $y_{i}=0$; otherwise employee $i$ has attained at most full recovery. Note that (1) and (2) are equivalent when employee I achieves exact full recovery, or when $a_{i} \Theta=b_{i} t$.

\subsection{Development of a Separable Quadratic Solution Procedure (SQSP)}

We are unaware of any readily available commercial code that will efficiently solve MBCP. Therefore, it was desirable to develop an easily implemented and efficient optimal solution procedure. To simplify 
procedural development, and without loss of generality, we impose an ordering on the employees such

that

$a_{n} / b_{n} \geqslant a_{n+1} / b_{n+1}$ for $1 \leqslant n<s$

where $S$ = the total number of employees in the work group. Any ties may be arbitrarily broken because it is the ratio $a_{n} / b_{n}$ that determines which of the five possible recovery conditions from subsection 2.1 are applicable for any given work-rest policy. With this ordering, it is possible to solve MBCP by first solving $s+1$ quadratic subproblems. The $(n+1)$ th subproblem (where $0 \leqslant n \leqslant s$ ) is:

$\operatorname{Max} Z_{n+1}(T)$

$=\sum_{i \in U_{n}} W A L F_{i}(T)+\sum_{i \in V_{n}} W A M F_{i}(T)$

subject to $b_{n} t-a_{n} \theta \leqslant 0$

$a_{n+1} \Theta-b_{n+1} t \leqslant 0$

and (8), (9), and (10), where $U_{n}=\{i \mid 1 \leqslant i \leqslant n\}$, and $V_{n}=\{i \mid b<i \leqslant s\}$.

Constraints 12 and 13 should be omitted in the first and last subproblems, respectively. The subproblem having the highest objective value provides the global optimal values of $\Theta^{*}$ and $t^{*}$.

\section{AN EFFICIENT METHOD FOR FINDING THE GLOBAL OPTIMAL GROUP WORK-REST POLICY}

In the subsections that follow, we first derive conditional optimal solutions to MBCP. The conditional solutions are then used to develop an efficient global optimal solution procedure (EOSP). Finally, we compare the relative computational efficiencies of SQSP and EOSP.

\subsection{Derivation of Model Properties}


In this subsection we show that if a break is taken, it should result in at least full recovery for one or more employees. We also develop optimal policies conditional on the nth employee experiencing exact and inexact full recovery. To reduce the complexity of subsequent equations, we will use the definitions:

$$
\begin{aligned}
& A=\sum_{i \in E} a_{i} \\
& B=\sum_{i \in E} b_{i} \\
& C=\sum_{i \in E} c_{i} \\
& A_{n}=\sum_{i \in V_{n}} a_{i} \\
& B_{n}=\sum_{i \in U_{n}} b_{i}
\end{aligned}
$$

\subsubsection{The Global Optimality of at Least Full Recovery for One or More Employees for Positive o}

Using the ordering in (10), the group work output with employees $1, \ldots, n$ experiencing at least full recovery and employees $n+1, \ldots, s$ experiencing at most full recovery is given by

$$
\begin{aligned}
& W_{n: n+1}(\Theta, t, T) \\
& =\sum_{i \in U_{n}} W A L F_{i}(T)+\sum_{i \in V_{n}} W A M F_{i}(T) .
\end{aligned}
$$

Lemma 1. For any fixed break duration $\Theta_{c}$ such that $T>\theta_{c}>0$, the global optimal policy results in one or more employees achieving at least full recovery.

Proof. Assume that the optimal policy results in no employee attaining at least full recovery. After simplification, the appropriate work function from (19) becomes $W_{0: 1}\left(\Theta_{C}, t, T\right)=-0.5\left[\left(T-\Theta_{C}\right)^{2} B\right]+\left(T-\Theta_{C}\right) C$ 


$$
+\left(T-t-\Theta_{c}\right) A \Theta_{c} .
$$

Since the first derivative of (20) with respect to t (the only variable in the equation) yields $-A \Theta_{c}$, the maximum of $W_{0: 1}\left(\Theta_{c}, t, T\right)$ is appropriate for $t \geqslant \theta a_{1} / b_{1}$, it is maximized when $t=a_{1} \Theta / b_{1}$, or when the break of length $\theta_{c}$ is placed such that full recovery occurs for the first employee. This contradicts our assumption that the optimal policy results in no one achieving at least full recovery and ends our proof of Lemma 1.

Given Lemma 1 and the ordering imposed in (10), if a positive global optimal value of $\Theta$ exists, there exists an employee $n \in E$ such that employees $n+1, \ldots, s$ are not fully recovered at the conclusion of the rest break and employee $n$ achieves either exact, or inexact full recovery. We will refer to these conditions as an exact full recovery strategy and as an inexact full recovery strategy, respectively. The next subsections develop optimal policies for these strategies.

\subsubsection{The Optimal Policy Conditional on an Exact Full Recovery Strategy for the $n$th Employee}

Defining $W_{n}(\Theta, T)$ as the work output obtained under an exact full recovery strategy for the $n$th employee and therefore substituting $t=\Theta a_{n} / b_{n}$ into (19) we obtain

$$
\begin{aligned}
& W_{n}(\Theta, T)=-0.5\left[(T-\theta)^{2} B\right]+(T-\theta) C \\
& \quad+\Theta\left[T-\Theta\left(1+a_{n} / b_{n}\right)\right]\left(a_{n} B_{n} / b_{n}+A_{n}\right) .
\end{aligned}
$$

Taking the first derivative of (21) with respect to $\Theta$ yields the conditional optimal value of $\Theta$

$\Theta_{n}^{*}=\frac{b_{n}^{2}\left[T\left(B+a_{n} B_{n} / b_{n}+A_{n}\right)-C\right]}{B b_{n}^{2}+2\left(a_{n}+b_{n}\right)\left(a_{n} B_{n}+b_{n} A_{n}\right)^{\prime}}$

where the conditional optimal value of $t$ is

$t_{n}^{*}=\Theta_{n}^{*} a_{n} / b_{n}$

The group work output given by $W_{n}\left(\Theta_{n}^{*}, T\right)$ is a local maximum because $d^{2} W_{n}(\Theta, T) / d \Theta^{2}<0$. 


\subsubsection{The Optimal Policy Conditional on an Inexact Full Recovery Strategy for the nth Employee}

Setting the first-order partial derivatives of $W_{n: n+1}(\Theta, t, T)$ as defined in (19) to zero, we obtain the critical point

$\Theta_{n: n+1}^{*}=\frac{2 B_{n}\left[T\left(B-B_{n} / 2+A_{n} / 2\right)-C\right]}{2 B_{n}\left(B+2 A_{n}\right)-\left(B_{n}+A_{n}\right)^{2}}$

and

$t_{n: n+1}^{*}=0.5\left[T-\Theta_{n: n+1}^{*}\left(1+A_{n} / B_{n}\right)\right]$.

For an inexact full recovery strategy to apply, however, the following strict inequalities must hold:

$b_{n} t_{n: n+1}^{*}<a_{n} \theta_{n: n+1}^{*}$, and

$b_{n+1} t_{n: n+1}^{*}>a_{n+1} \theta_{n: n+1}^{*}$.

Define $\left|H_{n: n+1}\right|$ as the determinant of the Hessian matrix of (19). Then

$\left|H_{n: n+1}\right|=2 B_{n}\left(B+2 A_{n}\right)-\left(B_{n}+A_{n}\right)^{2}$.

Since the second-order partial derivative of (19) with respect to $\Theta$ is negative, $W_{n: n+1}\left(\Theta_{n: n+1}^{*}, t_{n: n+1}^{*}, T\right)$ is a maximum if $\left|H_{n: n+1}\right|>0$.

\subsection{Global Optimal Group Work-Rest Policy Development}

In this subsection we develop the optimal group work-rest policies for short and long time horizons, respectively.

\subsubsection{The Global Optimal Work-Rest Policy for Short Time Horizons}

We demonstrated in subsection 3.1.1 that any fixed $\Theta>0$ should be positioned such that one or more employees should attain at least full recovery. However, we prove in this subsection that time horizons may exist that are too short to justify taking a rest break. We define a short time horizon as 
$T \leqslant C /(A+B)$.

Theorem 1. If $T$ is a short time horizon, then the global optimal policy is to take no rest break. That is, $\Theta^{*}=t^{*}=0$.

Proof. Begin with the optimal policies conditional on exact full recovery and define $S_{n}$ as the length of the time horizon for which $\Theta_{n}^{*}=0$. Then

$S_{n}=C /\left[B+a_{n} B_{n} / b_{n}+A_{n}\right]$.

Using the ordering imposed in (10) it is easy to show that $S_{s} \geqslant S_{s-1} \geqslant \cdots \geqslant S_{1}$. Given this latter ordering and (22), an optimal policy conditional on exact full recovery for the $n$th employee is only valid $\left(\Theta_{n}^{*}>0\right)$ if $T>S_{n} \geqslant S_{1}=C /\left[B+a_{1} / B_{1} / b_{1}+A_{1}\right]=C /(A+B)$.

Next consider the optimal policies conditional on inexact full recovery and define $S_{n: n+1}$ as the length of the time horizon for which the numerator of (24) is zero. Then

$S_{n: n+1}=C /\left[B-B_{n} / 2+A_{n} / 2\right]$.

Since we have assumed, as did Bechtold, Janaro and Sumners, that $a_{n} / b_{n}>0$ for $n \in E$, it is easy to show that $S_{n: n+1}>S_{n}$ for $n \in E$. Because the denominator of (24) is $\left|H_{n: n+1}\right|$, and because an optimal policy conditional on inexact full recovery for employee $n$ can only occur when $\left|H_{n: n+1}\right|>0$, positive values of $\Theta_{n: n+1}^{*}$ can occur only when the numerator of (24) is also positive. Hence, by the ordering of $S_{n}$ and $S_{n: n+1}, \Theta_{n: n+1}^{*}>0$ can only occur when $T>S_{n: n+1}>S_{n} \geqslant S_{1}=C /(A+B)$. Thus, if the time horizon is short, the global optimal policy is to take no break. This ends our proof of Theorem 1.

The group work output under a short time horizon, which we define as $W N B(T)$, is given by $W N B(T)=C T-0.5 T^{2} B$

\subsubsection{The Global Optimal Work-Rest Policy for Long Time Horizons}

We define a long time horizon as one that is not short. Thus, a long time horizon is in effect if $T>$ $C /(A+B)$. 
Corollary 1. If $T$ is a long time horizon, a positive duration rest-break should be scheduled.

Proof. Assuming that $\Theta_{n}^{*}>0$ it is easy to show that $W_{n}\left(\Theta_{n}^{*}, T\right)>W N B(T)$. From (30), $\Theta_{n}^{*}>0$ if and only if $S_{n}<T$. Thus, if $T>S_{1}=C /(A+B)$, then $\Theta_{1}^{*}>0$ and $W_{1}\left(\theta_{1}^{*}, T\right)>W N B(T)$. In other words, if $T$ is a long time horizon, group work output can be increased by scheduling a positive duration break. This completes our proof of Corollary 1.

The global optimal policy determination for a long time horizon is simply a search procedure.

The exact full recovery strategy yielding the greatest work output is found, and compared to the inexact full recovery strategy yielding the highest work output. Whichever is greater determines the global optimal policy.

Theorem 2. If $T$ is a long time horizon, a global optimal policy is:

$\Theta^{*}\left\{\begin{array}{c}\Theta_{n 1}^{*}, \text { if } W 1(T)>W 2(T) \\ \Theta_{n 2: n 2+1}^{*}, \text { otherwise, }\end{array}\right.$
$t^{*}\left\{\begin{array}{c}t_{n 1}^{*}, \text { if } W 1(T)>W 2(T) \\ t_{n 2: n 2+1}^{*}, \text { otherwise, }\end{array}\right.$

where

$$
\begin{aligned}
& N_{1}=\text { those employees } n \in E \text {, such that } S_{n}<T \text {; } \\
& N_{2}=\text { those employees } n \in E \text {, such that } S_{n: n+1}<T \text {, } \\
& \left|H_{n: n+1}\right|>0 \text {, and for which restrictions (26) and (27) hold; } \\
& W 1(T)=\max _{n \in N_{1}}\left\{W_{n}\left(\Theta_{n}^{*}, T\right)\right\} \\
& n_{1}=\text { any employee } n \in N_{1} \text { for which } \\
& W_{n}\left(\theta_{n}^{*}, T\right) \\
& W_{2}(T)=\left\{\max _{n \in N_{2}}\left\{W_{n: n+1}^{-\infty}\left(\Theta_{n: n+1}^{*}, t_{n: n+1}^{*}, T\right)\right\},\right. \\
& \text { otherwise; and } \\
& n_{2}=\text { any employee } n \in N_{2} \text { for which }
\end{aligned}
$$




$$
W_{n: n+1}\left(\Theta_{n: n+1}^{*}, t_{n: n+1}^{*}, T\right)=W 2(T) .
$$

Proof. Given that $T>C /(A+B)=S_{1}, N_{1}$ is not an empty set, and by Corollary $1, \theta^{*}>0$. By Lemma 1 , any global optimal policy that includes a rest period must result in the achievement of at least full recovery for one or more employees. Given that exact and inexact full recovery policies are valid only for $n \in N_{1}$ and $n \in N_{2}$, respectively, the global optimal policy must result in an optimal exact full recovery policy for employee $n_{t}$ if $W 1(T)>W 2(T)$, or an optimal inexact full recovery policy for employee $n_{2}$ otherwise.

\subsection{An Efficient Global Optimal Solution Procedure (EOSP)}

Once all employees have been rank ordered according to restriction (10), the following procedure identifies the global optimal solution: If $T \leqslant C /(A+B)$, then $\theta^{*}=t^{*}=0$. Otherwise, $\theta^{*}$ and $t^{*}$ are as specified in Theorem 2. We have assumed that all employees in the group have been rank ordered because this task must be completed whether solutions are obtained with EOSP or SQSP. Moreover, the computational effort of this task is likely to vary, depending upon the specific sort routine that is used.

It has been recognized that accounting for the existence of rest-break penalties is important in the development of multiple rest-break policies (Bechtold, Janaro and Sumners). Consider a time penalty $e>$ 0, which occurs at the beginning of any break period, where neither work nor recovery takes place. EOSP can easily be modified to account for this penalty by using $T_{e}=T-e$ in place of $T$.

\subsection{Computational Efficiency of EOSP}

Using an 80386-based personal computer we compared the time required to generate global optimal solutions using EOSP and SQSP. SQSP required three distinct steps for each of the $s+1$ subproblems: problem generation, solution, and interpretation of results. FORTRAN programs were developed for EOSP and also for the first and third steps of SQSP, while all quadratic subproblems associated with SQSP were solved using the QCP algorithm of LINDO-PC. Hypothetical sets of parameters were 
generated using uniform distributions with the following ranges: $0 \leqslant a_{i} \leqslant 0.01 ; 0 \leqslant b_{i} \leqslant$

0.01 ; and $0.8 \leqslant c_{i} \leqslant 1.2$. Based on two runs each for groups of 500, 1,000, and 2,000 employees, EOSP's solution time was a near-perfect 0.0006 seconds per employee in the work group. In contrast, SQSP required approximately 0.261 seconds per employee in the group, exclusive of the rank-ordering of employees, or more than 434 times the time required by EOSP. As EOSP is conceptually simple, easily implemented, and readily solved, it is likely to be of value in those environments meeting the assumptions of the model.

\section{THE PRODUCTIVITY IMPROVEMENT POTENTIAL OF MODEL-BASED POLICIES}

In this section we present the results of computer simulation experiments designed to investigate the output given by rest-break policies derived from the work characteristics of a random sample of employees, compared to the work output for a break policy determined using the work characteristics of all employees in the work group. Such an investigation is merited because decay and recovery rates are often not easily measured (Boothe 1984, Janaro and Bechtold 1985). We also briefly discuss the nature of optimal policy variables.

\subsection{Computer Simulation Parameters and Sampling Policies}

The efficacy of employee sampling policies was examined using simulated work groups. The experimental analysis was conducted within an overall structure consisting of four major factors: the mean levels of decay and recovery rates, work-group size, and the proportion of the work group to be sampled.

The parameter $T$ was fixed at 240 minutes to match the common work horizon of four hours within which a rest period is frequently inserted. A summary of employee work characteristics is presented in Table I. For each of the four major decay/recovery categories, 100 work groups were 
randomly generated for each of six different work-group sizes $(50,100,200,400,800,1,600)$. In each of the 2,400 runs, group rest-break policies and related work outputs were computed using the work characteristics of employees comprising $2 \%, 5 \%$, and $10 \%$ random samples, and compared to the work output possible when the rest-break policy was determined using the work characteristics of all employees in the group.

\subsection{Simulation Results}

A summary of relative work performance is displayed in Table II for all runs within the four decay and recovery categories. Policies based upon proportionately small samples were surprisingly effective. Although increasing the sample proportion generally resulted in improvements in both minimum and mean relative performance, the greatest impact was observed for minimum performance. For example, increasing the sample proportion from $2 \%$ to $5 \%$ improved the overall minimum relative performance from $98.25 \%$ to $99.27 \%$ in the fast decay/slow recovery category.

We report the mean policy variables that were derived from the model with $100 \%$ sampling in Table III. Global optimal rest-break policies varied substantially across the decay/recovery categories. On average, breaks were shorter and taken earlier in the slow decay category compared to the fast decay category, although optimal rest-break policies in all four categories exhibited the front loading observed in the optimal policies for individual employees across a diverse set of worker characteristics (Bechtold, Janaro and Sumners).

\section{DISCUSSION}

Our discussion is presented within two categories: limitations and future research; and conclusions.

INSERT TABLE I HERE

\footnotetext{
${ }^{a}$ The initial employee work rates spanned approximately $80 \%$ to $120 \%$ of standard, a performance rating range that could be expected in a variety of task environments (Barnes 1980). The range of the decay and
} 
recovery parameters also resulted in an approximate 50\% spread between the highest and lowest work decay rates and recovery-to-decay ratios, respectively.

\section{INSERT TABLE II HERE}

${ }^{a}$ Relative performances reported were based on $100 \mathrm{Ws} / \mathrm{Wp}$, where Ws = work output with employee sampling, and $\mathrm{Wp}=$ work output with the policy determined based on the work characteristics of all employees in the work group.

${ }^{b} \mathrm{~F}$ = fast; $\mathrm{S}=$ slow; $\mathrm{D}$ = decay; $\mathrm{R}$ = recovery.

\section{INSERT TABLE III HERE}

\footnotetext{
${ }^{a}$ As determined using EOSP based on the work characteristics of all employees in the work group. ${ }^{b} n=$ the number of simulation runs (out of a possible 600) with $\theta^{*}>0 ; \theta_{a}=$ the mean length of the optimal rest period; $t_{a}=$ the mean length of the optimal initial work period.
}

\subsection{Limitations and Future Research}

The limitations of the current research underscore several issues worthy of additional research. We first address two potential limitations associated with the simulation experiment, and next consider the possible implications of the assumptions upon which the current model was based.

First, the simulation approach reported here could be used to investigate the behavior of optimal policies for other distributions of employee characteristics. In particular, how sensitive are the policies to differences in employee characteristics? Can useful general guidelines be developed to avoid costly and difficult measurement procedures?

Second, we offer the caveat that we assumed no measurement error in the determination of employee work characteristics. Although the effect of measurement error is an interesting issue, the effectiveness of the sampling policies suggests that it is not likely to be a problem. It may be likely that greater accuracy would be more important, relative to group productivity, for smaller groups of employees with high variances in performance characteristics.

There are several potential research questions that result from our modeling assumptions. Although evidence exists that work decay and work recovery are linear (Boothe 1984, Janaro and 
Bechtold 1985), it is doubtful that these work characteristics would be linear for all employees across a broad range of tasks. How effective are policies based upon linear models for task environments where the decay and/or recovery rates of one or more employees in the work group are nonlinear? Bechtold and Sumners found for individual employees that using policies derived from linear models when decay was exponential produced acceptable results only when rates of decay and recovery were relatively slow. The effectiveness of sampling-based policies is also called into question when the nature of the decay and recovery characteristics for the work group are unknown. These issues could perhaps be resolved through validation of the model using real-world employee work groups.

Multiple rest periods have been shown to be effective for individuals (Boothe 1984, Janaro and Bechtold 1985). It is possible that multibreak models for employee groups also may be beneficial.

With a single exception (Bechtold 1991), all previously published work-rest modeling research, and most of the related empirical research, has considered only a single planning horizon. Policy development that integrates two work stretches (separated by a meal break or arising from split shifts) in the work group environment would be of potential value. In addition, it may be worthwhile to consider the productivity impact of including the time horizon $T$ as an endogenous variable.

Workers have been observed to exhibit a warm-up period before reaching their maximum work rate. When this occurs with an individual employee working on an individual task, the solution is to input a revised time horizon to the model that is equal to the length of the work stretch available less the duration of the warm-up period (Bechtold, Janaro and Sumners). With groups of employees, there is no obvious simple solution since warm-up periods may vary across employees.

Finally, the difference in optimal rest-break policies across decay/recovery categories, and the great divergence of the optimal policies from the traditional policy of a 10-15 minute break taken approximately midway through a 4-hour work stretch, deserve attention. Conducting empirical investigations to measure employee decay and recovery characteristics in a broad range of operating environments would thus be warranted before performing further simulation analyses. 


\subsection{Conclusions}

This research has extended work-rest modeling from individual employees to groups of employees. As published reports of implementation and evaluation of previous research results have been scant, this expanded framework should not only provide motivation for further research within on-going organizations, but should also provide a stimulus for greater integration of modeling and empirical research. Specifically, laboratory or field-based experiments to validate the model have the potential to provide new insights into a research stream that, historically, has been based upon empirical search for effective work-rest cycles. 


\section{ACKNOWLEDGMENT}

We are grateful to Professor Michael Magazine, an anonymous associate editor, and two referees for their helpful comments.

\section{REFERENCES}

Aquilano, N.J. 1968. Work Physiology: A Physiological Evaluation of Time Standards and Work-Rest Design for Moderate to Strenuous Work. Doctoral Dissertation, University of California.

Dissertation Abstracts International, 29, 997A-1983A, University Microfilms No. 68-16, 508, Ann Arbor, Michigan.

Baker, M.A.,D.H. Holding and M. Loeb. 1984. Noise, Sex and Time of Day Effects in a Mathematical Task. Ergonomics 27, 67-80.

Barnes, R.M. 1980. Motion and Time Study. John Wiley, New York.

Bechtold, S.E. 1979. Quantitative Models for Optimal Rest Period Scheduling: A Note. Omega 7, 565566.

Bechtold, S.E. 1991. Optimal Work-Rest Schedules With a Set of Fixed-Duration Rest Periods. Dec. Sci. 22, 157-170.

Bechtold, S.E., and D.L. Sumners. 1988. Optimal Work-Rest Scheduling With Exponential Work-Rate Decay. Mgmt. Sci. 34, 547-552.

Bechtold, S.E., R.E. Janaro and D.L. Sumners. 1984. Maximization of Labor Productivity Through Optimal Rest-Break Schedules. Mgmt. Sci. 30, 1442-1458.

Boothe, R.S. 1984. Optimization of Rest Breaks: A Productivity Enhancement. Dissertation, Florida State University. University Microfilms International No. 842290, Ann Arbor, Michigan.

Eilon, S. 1964. On a Mechanistic Approach to Fatigue and Rest Periods. Int. J. Prod. Res. 3, 327-332.

Gentzler, G.L., T.M. Khalil and B.D. Sivazlian. 1977. Quantitative Models for Optimal Rest Period Scheduling. Omega 5, 215-220. 
Hagan, S.J., R.H. Wilkerson and C.E. Noble. 1980. Pursuit Tracking Skill as a Joint Function of Work and Rest Variables. Perceptual and Motor Skills 50, 683-697.

Janaro, R. E., a n d S. E. Bechtold. 1985. A Study of the Reduction of Fatigue Impact on Productivity Through Optimal Rest Break Scheduling. Human Factors 27, 459-466.

Lee, F.S. 1974. The Human Machine and Industrial Efficiency. Hive, Easton, Maryland.

Levine, J.M., T. Romashko and E.A. Fleishman. 1973. Evaluation of an Abilities Classification System for Integrating and Generalizing Human Performance Research Findings: An Application to Vigilance Tasks. J. Applied Psych. 58, 149-157.

McCormack, P.D. 1958. Performance in a Vigilance Task as a Function of Inter-Stimulus Interval and Interpolated Rest. Canadian J. Psych. 12, 242-246.

McFarland, R.A. 1971. Understanding Fatigue in Modem Life. Ergonomics 14, 1-10.

Morgan, B. B., and E. W. Pitts. 1985. Methodological Issues in the Assessment of Sustained Performance. Behav. Res. Meth. Instrum. Comput. 17, 96-101.

Murrell, K.F.H. 1965. Human Performance in Industry. Reinhold Publishing, New York. Okogbaa, O. G. 1983. An Empirical Model for Mental Work Output and Fatigue. Doctoral Dissertation, University of Cincinnati. University Microfilms No. 8323227, Ann Arbor, Michigan.

Woodward, D.P., and P.D. Nelson. 1974. A User Oriented Review of the Literature on the Effects of Sleep Loss, Work-Rest Schedules, and Recovery Performance. Office of Naval Research, Washington, D.C. 
Figure 1.

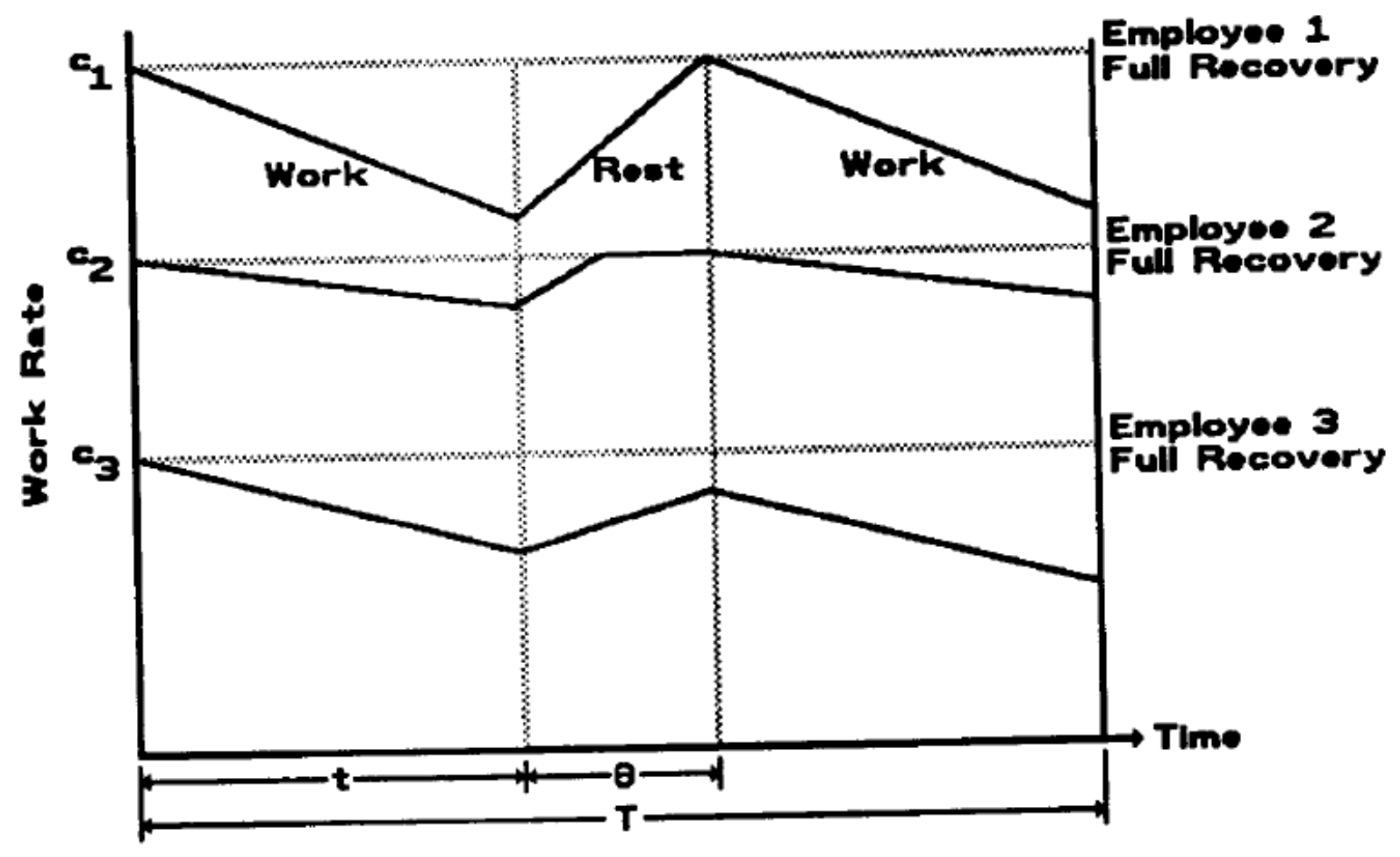

Table I

Summary of Employee Work Characteristics in the Simulation Experiments

\begin{tabular}{|c|c|c|c|c|}
\hline Parameter & $\begin{array}{l}\text { Range in the Mean } \\
\text { Within-Run } \\
\text { Parameter Values } \\
\text { Across Runs } \\
\end{array}$ & $\begin{array}{c}\text { Distribution, Across } \\
\text { Runs, of the } \\
\text { Mean Within-Run } \\
\text { Parameter Value }\end{array}$ & $\begin{array}{l}\text { Standard Deviation of the } \\
\text { Parameters Within Each Run }\end{array}$ & $\begin{array}{c}\text { Distribution of } \\
\text { the Parameters } \\
\text { Within Each } \\
\text { Run }\end{array}$ \\
\hline $\mathrm{c}$ & $1.0-1.0$ & NA & 0.067 & normal \\
\hline b (slow decay) & $0.000208-0.002083$ & uniform & $1 / 15$ of the mean decay rate for the run & normal \\
\hline b (fast decay) & $0.002083-0.003958$ & uniform & $1 / 15$ of the mean decay rate for the run & normal \\
\hline a (slow recovery) & $\begin{array}{l}10 \%-200 \% \text { of the } \\
\text { mean decay rate } \\
\text { for the run }\end{array}$ & uniform & $1 / 15$ of the mean recovery-to-decay ratio for the run & normal \\
\hline a (fast recovery) & $\begin{array}{l}200 \%-390 \% \text { of the } \\
\text { mean decay rate } \\
\text { for the run }\end{array}$ & uniform & $1 / 15$ of the mean recovery-to-decay ratio for the run & normal \\
\hline
\end{tabular}




\section{Table II}

Relative Performance of Sampling Policies Versus the Perfect Knowledge-Based Policy ${ }^{a}$

\begin{tabular}{|c|c|c|c|c|c|}
\hline \multirow{2}{*}{$\begin{array}{c}\text { Percentage of } \\
\text { the Work } \\
\text { Group Sampled }\end{array}$} & \multirow[b]{2}{*}{ Statistic } & \multicolumn{4}{|c|}{ Decay/Recovery Category $^{b}$} \\
\hline & & SD/SR & $\mathrm{SD} / \mathrm{FR}$ & FD/SR & FD/FR \\
\hline 2 & $\begin{array}{l}\text { Minimum } \\
\text { Mean }\end{array}$ & $\begin{array}{l}99.3651 \\
99.9987\end{array}$ & $\begin{array}{l}99.4352 \\
99.9936\end{array}$ & $\begin{array}{l}98.2544 \\
99.9728\end{array}$ & $\begin{array}{l}98.8406 \\
99.9684\end{array}$ \\
\hline 5 & $\begin{array}{l}\text { Minimum } \\
\text { Mean }\end{array}$ & $\begin{array}{l}99.8217 \\
99.9993\end{array}$ & $\begin{array}{l}99.7923 \\
99.9975\end{array}$ & $\begin{array}{l}99.2729 \\
99.9883\end{array}$ & $\begin{array}{l}99.2948 \\
99.9875\end{array}$ \\
\hline 10 & $\begin{array}{l}\text { Minimum } \\
\text { Mean }\end{array}$ & $\begin{array}{l}99.8504 \\
99.9998\end{array}$ & $\begin{array}{l}99.8586 \\
99.9986\end{array}$ & $\begin{array}{l}99.5593 \\
99.9942\end{array}$ & $\begin{array}{l}99.7134 \\
99.9931\end{array}$ \\
\hline
\end{tabular}

\section{Table III \\ Optimal Policy Variables ${ }^{a}$}

Decay/Recovery Category

\begin{tabular}{ccccc}
\cline { 2 - 5 } Variable $^{b}$ & SD/SR & SD/FR & FD/SR & FD/FR \\
\hline$n$ & 56 & 297 & 489 & 600 \\
$\Theta_{a}$ & 0.74 & 5.71 & 22.38 & 24.69 \\
$t_{a}$ & 1.35 & 18.45 & 29.67 & 71.08 \\
\hline
\end{tabular}

